\title{
Existence and Uniqueness of Positive Solitons for a Second-Order Difference Equation
}

\author{
Xinfu Li, Guang Zhang, and Ying Wang \\ School of Science, Tianjin University of Commerce, Tianjin 300134, China \\ Correspondence should be addressed to Guang Zhang; qd_gzhang@126.com
}

Received 11 September 2014; Revised 12 November 2014; Accepted 15 November 2014; Published 2 December 2014

Academic Editor: Juan J. Nieto

Copyright (C) 2014 Xinfu Li et al. This is an open access article distributed under the Creative Commons Attribution License, which permits unrestricted use, distribution, and reproduction in any medium, provided the original work is properly cited.

A discrete logistic steady-state equation with both positive and negative birth rate of population will be considered. By using suband upper-solution method, the existence of bounded positive solutions and the existence and uniqueness of positive solitons will be established. To this end, the Dirichlet eigenvalue problem with positive and negative coefficients is considered, and a general sub- and upper-solution theorem is also obtained.

\section{Introduction}

In this paper, we consider the second-order difference equation

$$
-\Delta^{2} x_{i-1}=\lambda\left(p_{i} x_{i}-x_{i}^{1+\alpha}\right), \quad i \in \mathbb{Z},
$$

where $\mathbb{Z}$ is a set of all integers, $\alpha>0$ and $\lambda>0$ are constants, $\Delta x_{i}=x_{i+1}-x_{i}, \Delta^{2} x_{i-1}=\Delta\left(\Delta x_{i-1}\right)$, and $\left\{p_{i}\right\}_{i \in \mathbb{Z}}$ will be specified later.

This equation is associated with the famous discrete logistic equation with diffusion:

$$
x_{i}^{t+1}-x_{i}^{t}=d \Delta^{2} x_{i-1}^{t}+p_{i} x_{i}^{t}-\left(x_{i}^{t}\right)^{1+\alpha}
$$

for $i \in \mathbb{Z}$ and $t \in \mathbb{Z}^{+}=\{0,1,2, \ldots\}$, where the parameter $d>0$ corresponds to the rate at which the population diffuses, and the unknown function $x$ corresponds to the density of a population. The term $-x^{1+\alpha}$ in the equation corresponds to the fact that the population is self-limiting and the $p$ corresponds to the birth rate of population if the self-limitation is ignored. At points where $p_{i}>0(<0)$ the population, ignoring self-limitation, has positive (negative) birth rate. Hence, we assume throughout this paper that $p$ takes on both positive and negative values on $\mathbb{Z}$; we further assume that the sequence $\left\{p_{i}\right\}_{i \in \mathbb{Z}}$ is bounded and there exists an integer $i_{0} \in \mathbb{Z}$ such that $p_{i_{0}}>0$.
If we write $\lambda=1 / d$, we see that the steady-state solutions of (2) must satisfy (1). Since the sequence $x$ represents a population density, it is nonnegative. Such solutions correspond to possible steady-state distributions of population. So our purpose in this paper is to establish the existence of positive solutions or the existence and uniqueness of positive solitons for (1). A discrete soliton is a spatially localized solution of (1) and decays to 0 at infinity; that is,

$$
\lim _{|i| \rightarrow \infty} x_{i}=0 .
$$

Recently, the existence of nontrivial discrete solitons for the general equation

$$
-\Delta^{2} x_{i-1}=f\left(i, x_{i}\right), \quad i \in \mathbb{Z},
$$

has been extensively established by a number of authors [112]. Among the methods used are the principle of anticontinuity [1-3], variational methods [4-10], center manifold reduction [11], Nehari manifold approach [12], and so forth. On the other hand, a soliton of (4) is also its homoclinic orbit or homoclinic solution. By using the variational methods, the existence of homoclinic orbits or homoclinic solutions has also been extensively discussed by a number of authors; see $[4-10,13-16]$. However, as far as we know, there are few papers concerned with the existence of positive solitons. 
Equation (2) is a discrete analogue of the well-known logistic equation of the form

$$
u_{t}=D u_{x x}+p(x) u-u^{1+\alpha}, \quad x \in \mathbb{R}, t \in \mathbb{R}^{+} .
$$

Indeed, by means of standard finite difference methods, we set up a grid in the $x, t$ plane with grid spacings $\Delta x$ and $\Delta t$ and then replace the second derivative $u_{x x}$ with a central difference and $u_{t}$ with a forward difference. By writing $x_{i}=$ $i \Delta x, t_{n}=n \Delta t, p_{i}=p\left(x_{i}\right)$, and $u_{i}^{n} \approx u\left(x_{i}, t_{n}\right)$, a finite difference scheme for (5) is obtained:

$$
\begin{aligned}
& \frac{u_{i}^{n+1}-u_{i}^{n}}{\Delta t} \\
& \quad=\frac{D}{(\Delta x)^{2}}\left(u_{i+1}^{n}-2 u_{i}^{n}+u_{i-1}^{n}\right)+p_{i} u_{i}^{n}-\left(u_{i}^{n}\right)^{1+\alpha}
\end{aligned}
$$

or

$$
\begin{aligned}
& u_{i}^{n+1}-u_{i}^{n} \\
& \quad=\frac{D \Delta t}{(\Delta x)^{2}}\left(u_{i+1}^{n}-2 u_{i}^{n}+u_{i-1}^{n}\right)+\Delta t p_{i} u_{i}^{n}-\Delta t\left(u_{i}^{n}\right)^{1+\alpha},
\end{aligned}
$$

which has the steady-state equation:

$$
-\Delta^{2} x_{i-1}=\frac{(\Delta x)^{2}}{D}\left(p_{i} x_{i}-x_{i}^{1+\alpha}\right), \quad i \in \mathbb{Z} .
$$

We note that the steady-state equation of (5) is

$$
D u^{\prime \prime}+p(x) u-u^{1+\alpha}=0
$$

or

$$
-u^{\prime \prime}=\mu\left(p(x) u-u^{1+\alpha}\right),
$$

where $\mu=1 / D$. When $\alpha=1,(10)$ is reduced to

$$
-u^{\prime \prime}=\mu(p(x) u-u) .
$$

In [17], the authors considered the existence and uniqueness of positive solitons of (11) by using sub- and upper-solution method. The present work is motivated by [17].

To obtain a positive subsolution of (1), we need a positive eigenvalue and its corresponding positive eigenfunction of the eigenvalue problem

$$
\begin{gathered}
-\Delta^{2} x_{i-1}=\lambda p_{i} x_{i}, \quad i \in\{a, a+1, \ldots, b\}, \\
x_{a-1}=0=x_{b+1} .
\end{gathered}
$$

Note that $p$ takes on both positive and negative values; thus, it is indefinite in a very strong sense. The corresponding problem for ordinary differential equation had been considered in [18] by using variational method. However, such problem is new for the above discrete problem; see [19-21]. In Section 2, we will consider the above discrete eigenvalue problem by using the matrix and vector method.

As far as we know, for the discrete problem (1) there is no general sub- and upper-solution theorem on the set
$\mathbb{Z}$. Thus, a general sub- and upper-solution theorem will be firstly obtained in Section 3 and such theorem will be used for (1). On the other hand, our solutions are classical; however, [17] cannot insure this fact because some points of their subsolution are not derivative.

Our results will give some theory groundwork for the numerical calculation of (11). Note that $\lambda=(\Delta x)^{2} / D \neq \mu$ when $(\Delta x)^{2} \neq 1$. This will lead to different results between (1) and (11).

\section{Preliminaries}

For any $a, b \in \mathbb{Z}$ with $a<b$, we consider the eigenvalue problem of the form

$$
\begin{gathered}
-\Delta^{2} x_{i-1}=\lambda p_{i} x_{i}, \quad i \in[a, b], \\
x_{a-1}=0=x_{b+1},
\end{gathered}
$$

where $[a, b]=\{a, a+1, \ldots, b\},\left\{p_{i}\right\}_{i=a}^{b}$ is a real sequence, and there exists $i_{0} \in[a, b]$ such that $p_{i_{0}}>0$.

Denote

$$
x=\left(\begin{array}{c}
x_{a} \\
x_{a+1} \\
\vdots \\
x_{b}
\end{array}\right),
$$

$$
\begin{aligned}
A & =\left(\begin{array}{ccccc}
2 & -1 & 0 & \cdots & 0 \\
-1 & 2 & -1 & \cdots & 0 \\
& \cdots & \cdots & \cdots & \\
0 & \cdots & & 2 & -1 \\
0 & \cdots & 0 & -1 & 2
\end{array}\right)_{(b-a+1) \times(b-a+1)}, \\
P & =\left(\begin{array}{cccc}
p_{a} & 0 & \cdots & 0 \\
0 & p_{a+1} & \cdots & 0 \\
& \cdots & \cdots & \\
0 & \cdots & 0 & p_{b}
\end{array}\right)_{(b-a+1) \times(b-a+1)}
\end{aligned}
$$

Then problem (13) can be rewritten by matrix and vector in the form

$$
A x=\lambda P x .
$$

Let $H$ be a set of all real sequences $\left\{x_{i}\right\}_{i=a-1}^{b+1}$ with $x_{a-1}=$ $0=x_{b+1}$. For any $u, v \in H$, the inner product is defined as $(u, v)=\sum_{i=a}^{b} u_{i} v_{i}$ and the norm $\|\cdot\|$ is defined as $\|u\|=$ $\sqrt{\sum_{i=a}^{b} u_{i}^{2}}$.

For any $v \in H$, we define

$$
Q_{\lambda}(v)=v^{T} A v-\lambda \sum_{i=a}^{b} p_{i} v_{i}^{2}
$$

Consider the Rayleigh quotient

$$
K(v)=\frac{v^{T} A v}{\sum_{i=a}^{b} p_{i} v_{i}^{2}}
$$


and let

$$
\lambda_{1}=\inf \left\{K(v): v \in H, \sum_{i=a}^{b} p_{i} v_{i}^{2}>0\right\} .
$$
result.

For such defined $\lambda_{1}$, we have the following important

Lemma 1. $\lambda_{1}$ is a positive eigenvalue of the problem (13) or (16). Moreover $\lambda_{1}$ is simple and the corresponding eigenfunction $\varphi$ can be chosen such that $\varphi_{i}>0$ for all $i \in[a, b]$.

Proof. First, we show $\lambda_{1}>0$. Clearly

$$
Q_{\lambda_{1}}(v)=v^{T} A v-\lambda_{1} \sum_{i=a}^{b} p_{i} v_{i}^{2} \geq 0
$$

for all $v \in H$. Moreover, by the spectral theorem, $(A v, v) \geq$ $\gamma_{1}(v, v)$, where

$$
\gamma_{1}=4 \sin ^{2} \frac{\pi}{2(b-a+2)}
$$

is the first eigenvalue of the eigenvalue problem

$$
\begin{gathered}
-\Delta^{2} x_{i-1}=\gamma x_{i}, \quad i \in[a, b], \\
x_{a-1}=0=x_{b+1} .
\end{gathered}
$$

Hence, if $v \in H$ and $\sum_{i=a}^{b} p_{i} v_{i}^{2}>0$, then

$$
\begin{aligned}
K(v) & =\frac{v^{T} A v}{\sum_{i=a}^{b} p_{i} v_{i}^{2}} \geq \frac{\gamma_{1}(v, v)}{\sum_{i=a}^{b} p_{i} v_{i}^{2}} \\
& \geq \frac{\gamma_{1}}{\max \left\{\left|p_{i}\right|, i=a, a+1, \ldots, b\right\}}>0 .
\end{aligned}
$$

Hence $\lambda_{1}>0$.

Second, consider the linear eigenvalue problem

$$
\begin{gathered}
-\Delta^{2} u_{i-1}-\lambda_{1} p_{i} u_{i}=\mu u_{i}, \quad i \in[a, b], \\
u_{a-1}=0=u_{b+1},
\end{gathered}
$$

and define $(S u)_{i}=-\Delta^{2} u_{i-1}-\lambda_{1} p_{i} u_{i}$. It is easy to see that $\lambda_{1}$ is an eigenvalue for (13) with corresponding eigenfunction $\varphi$ if and only if 0 is an eigenvalue of $S$ and so of (24) with corresponding eigenfunction $\varphi$. The minimal eigenvalue $\alpha_{1}$ of $S$ is given by

$$
\begin{aligned}
\alpha_{1} & =\inf \left\{v^{T} A v-\lambda_{1} \sum_{i=a}^{b} p_{i} v_{i}^{2}: v \in H,\|v\|=1\right\} \\
& =\inf \left\{Q_{\lambda_{1}}(v): v \in H,\|v\|=1\right\} .
\end{aligned}
$$

Note that $Q_{\lambda_{1}}(v) \geq 0$ for all $v \in H$; thus, we have $\alpha_{1} \geq 0$. Because of how we defined $\lambda_{1}$, there exists a sequence $v^{(n)} \in$ $H$ and $\sum p_{i}\left(v_{i}^{(n)}\right)^{2}>0$ such that

$$
\lim _{n \rightarrow \infty} \frac{\left(v^{(n)}\right)^{T} A v^{(n)}}{\sum_{i=a}^{b} p_{i}\left(v_{i}^{(n)}\right)^{2}}=\lambda_{1}
$$

which implies that

$$
\lim _{n \rightarrow \infty} Q_{\lambda_{1}}\left(v^{(n)}\right)=0 .
$$

Thus, we have $\alpha_{1} \leq 0$. In this case, we know that $\alpha_{1}=0$ is the minimal eigenvalue of (24). By Lemma A.1 in the Appendix, $\alpha_{1}$ is simple and the corresponding eigenfunction can be chosen to be positive on $H$. Thus, the statement in this lemma is right.

Let $\lambda>\lambda_{1}$; consider the eigenvalue problem of the form

$$
\begin{gathered}
-\Delta^{2} u_{i-1}-\lambda p_{i} u_{i}=\mu u_{i}, \quad i \in[a, b], \\
u_{a-1}=0=u_{b+1} .
\end{gathered}
$$

We have the following result.

Lemma 2. If $\lambda>\lambda_{1}$, then the minimal eigenvalue $\mu_{1}$ of (28) is negative and the corresponding eigenfunction $\varphi^{(1)}$ can be chosen so that $\varphi_{i}^{(1)}>0$ for all $i \in[a, b]$.

Proof. Let $\varphi$ be the eigenvector obtained in Lemma 1 and $\|\varphi\|=1$; then we have

$$
\begin{aligned}
\varphi^{T} A \varphi-\lambda \sum_{i=a}^{b} p_{i} \varphi_{i}^{2} & =\frac{\lambda}{\lambda_{1}} \varphi^{T}\left(A-\lambda_{1} P\right) \varphi-\frac{\lambda-\lambda_{1}}{\lambda_{1}} \varphi^{T} A \varphi \\
& =-\frac{\lambda-\lambda_{1}}{\lambda_{1}} \varphi^{T} A \varphi<0 .
\end{aligned}
$$

Consequently,

$$
\mu_{1}=\min \left\{\psi^{T} A \psi-\lambda \sum_{i=a}^{b} p_{i} \psi_{i}^{2} \mid\|\psi\|=1\right\}<0 .
$$

In view of Lemma A.1 in the Appendix, the eigenvector corresponding to $\mu_{1}$ can be chosen to be positive. This completes the proof.

\section{Main Results}

First of all, we introduce the definitions of the subsolution and upper-solution and give a general sub- and upper-solution theorem.

For any $a, b \in \mathbb{Z}$ with $a<b$, consider the Dirichlet boundary value problem of the form

$$
\begin{gathered}
-\Delta^{2} x_{i-1}=f\left(i, x_{i}\right) \quad \text { for } i \in[a, b], \\
x_{a-1}=0=x_{b+1} .
\end{gathered}
$$

We will denote by (31) ${ }_{n}$ the problem (31) when $a=-n$ and $b=n$.

Definition 3. A sequence $\bar{x}=\left\{\bar{x}_{i}\right\}_{i=a-1}^{b+1}$ is said to be an uppersolution of (31) if

$$
\begin{gathered}
-\Delta^{2} \bar{x}_{i-1} \geq f\left(i, \bar{x}_{i}\right) \quad \text { for } i \in[a, b], \\
\bar{x}_{i} \geq 0 \text { for } i=a-1, b+1 .
\end{gathered}
$$


A sequence $\underline{x}=\left\{\underline{x}_{i}\right\}_{i=-\infty}^{+}$is said to be a subsolution of (31) if

$$
\begin{gathered}
-\Delta^{2} \underline{x}_{i-1} \leq f\left(i, \underline{x}_{i}\right) \quad \text { for } i \in[a, b], \\
\underline{x}_{i} \leq 0 \text { for } i=a-1, b+1 .
\end{gathered}
$$

Theorem 4. Suppose that $\underline{x}, \bar{x}: \mathbb{Z} \rightarrow \mathbb{R}$ are functions with $\underline{x} \leq \bar{x}$ (where $\underline{x} \leq \bar{x}$ is defined by $\underline{x}_{i} \leq \bar{x}_{i}$ for $i \in \mathbb{Z}$ ) and $\left.\underline{x}\right|_{n},\left.\bar{x}\right|_{n}$ are, respectively, a subsolution and an upper-solution of (31) for all large $n$. Assume that $f(i, z)$ is continuous with $z \in[\alpha, \beta]$ and there exists a constant $k>0$ such that

$$
f\left(i, s_{2}\right)-f\left(i, s_{1}\right) \geq-k\left(s_{2}-s_{1}\right)
$$

for all $i \in \mathbb{Z}$ and $s_{2}>s_{1}$ with $s_{1}, s_{2} \in[\alpha, \beta]$ (where $\alpha=\inf _{i \in \mathbb{Z}} \underline{x}_{i}$ and $\beta=\sup _{i \in \mathbb{Z}} \bar{x}_{i}$ ). Then problem (4) has a solution $x$ such that $x \leq x \leq \bar{x}$.

Proof. Since $\underline{x}$ and $\bar{x}$ provide sub- and upper-solutions for $(31)_{n}$, there exists a solution $\varphi$ of $(31)_{n}$ such that $\underline{x}_{i} \leq \varphi_{i} \leq \bar{x}_{i}$ for all $|i| \leq n$.

In fact, for any sequence $u=\left\{u_{i}\right\}_{i=-n}^{n}$, clearly, the problem

$$
\begin{gathered}
-\Delta^{2} w_{i-1}+k w_{i}=f\left(i, u_{i}\right)+k u_{i} \text { for } i \in[-n, n], \\
w_{-n-1}=0=w_{n+1},
\end{gathered}
$$

has a unique solution $w$. This defines a mapping $T: u \rightarrow$ $w$. We claim that $T$ is increasing on $[\underline{x}, \bar{x}]$. In fact, for any sequences $\left\{\widetilde{u}_{i}\right\}_{i=-n}^{n},\left\{\widetilde{v}_{i}\right\}_{i=-n}^{n} \in[\underline{x}, \bar{x}]$ with $\widetilde{v} \leq \widetilde{u}$, we have

$$
\begin{aligned}
\Gamma(T \widetilde{u}-T \widetilde{v}) & =f\left(i, \tilde{u}_{i}\right)+k \tilde{u}_{i}-f\left(i, \widetilde{v}_{i}\right)-k \widetilde{v}_{i} \\
& \geq-k\left(\widetilde{u}_{i}-\widetilde{v}_{i}\right)+k\left(\widetilde{u}_{i}-\widetilde{v}_{i}\right) \geq 0,
\end{aligned}
$$

where $\Gamma$ is an operator defined as $(\Gamma u)_{i}=-\Delta^{2} u_{i-1}+k u_{i}$ for $i \epsilon$ $[-n, n]$. Since $(T \widetilde{u})_{-n-1}-(T \widetilde{v})_{-n-1} \geq 0,(T \widetilde{u})_{n+1}-(T \widetilde{v})_{n+1} \geq 0$, by the strong monotonicity of $\Gamma$, we obtain $T \widetilde{u} \geq T \widetilde{v}$; see [21].

Let $u^{(m)}=T u^{(m-1)}, u^{(0)}=\underline{x} ; v^{(m)}=T v^{(m-1)}, v^{(0)}=\bar{x}$. In the following, we claim that

$$
\underline{x}=u^{(0)} \leq u^{(1)} \leq u^{(2)} \leq \cdots \leq v^{(2)} \leq v^{(1)} \leq v^{(0)}=\bar{x} .
$$

First of all, by using (35) and the definition of subsolution, we see

$$
\begin{aligned}
-\Delta^{2} & \left(u_{i-1}^{(0)}-u_{i-1}^{(1)}\right)+k\left(u_{i}^{(0)}-u_{i}^{(1)}\right) \\
& =-\Delta^{2} u_{i-1}^{(0)}+k u_{i}^{(0)}-\left[f\left(i, u_{i}^{(0)}\right)+k u_{i}^{(0)}\right] \\
& =-\Delta^{2} \underline{x}_{i-1}-f\left(i, \underline{x}_{i}\right) \leq 0 \text { for } i \in[-n, n],
\end{aligned}
$$

and $u_{-n-1}^{(0)}-u_{-n-1}^{(1)} \leq 0, u_{n+1}^{(0)}-u_{n+1}^{(1)} \leq 0$. This implies that $u^{(0)}-u^{(1)} \leq 0$ by the strong monotonicity of $\Gamma$. A similar argument gives $v^{(1)} \leq v^{(0)}$. The monotonicity of $\Gamma$ and $T$ gives the rest. So there exist $u$ and $v$ such that

$$
\lim _{m \rightarrow \infty} u^{(m)}=u, \quad \lim _{m \rightarrow \infty} v^{(m)}=v .
$$

By the definition of $u$ and $v$, we see that $u$ and $v$ satisfy (31) ${ }_{n}$ and $\underline{x} \leq u \leq v \leq \bar{x}$. Let $x^{(n)}$ denote such solution corresponding to $(31)_{n}$.
Then standard a priori estimates and a diagonalization argument show that there exists a subsequence of $\left\{x^{(n)}\right\}$ which converges to solution $x$ of (4) on every bounded subset of $\mathbb{Z}$. Moreover, since $\underline{x} \leq x^{(n)} \leq \bar{x}$ for all $n$, it follows that $\underline{x} \leq x \leq \bar{x}$ on $\mathbb{Z}$. The proof is complete.

In the following, we use the sub- and upper-solution theorem to establish the existence of positive solutions or the existence and uniqueness of positive solitons for (1).

For problem (1), we have assumed that there exists $i_{0} \in \mathbb{Z}$ such that $p_{i_{0}}>0$. For any $n>\left|i_{0}\right|$, consider the eigenvalue problem (13) when $a=-n$ and $b=n$ and denote the corresponding positive eigenvalue $\lambda_{1}$ defined in (19) by $\lambda_{1}^{(n)}$. Then

$$
\lambda_{1}^{(\infty)}:=\lim _{n \rightarrow \infty} \lambda_{1}^{(n)} \geq 0
$$

Theorem 5. For any $\lambda>\lambda_{1}^{(\infty)}$, (1) has a positive solution, where $\lambda_{1}^{(\infty)}$ is defined in (40).

Proof. For any $\lambda>\lambda_{1}^{(\infty)}$, clearly, there exists a positive integer $n_{1} \geq\left|i_{0}\right|$ such that $i_{0} \in\left[-n_{1}, n_{1}\right]$ and $\lambda_{1}^{(\infty)}<\lambda_{1}^{\left(n_{1}\right)}<\lambda$. In view of Lemma 2, the eigenvalue problem

$$
\begin{aligned}
-\Delta^{2} x_{i-1}-\lambda p_{i} x_{i} & =\mu x_{i}, \quad i \in\left[-n_{1}, n_{1}\right], \\
x_{-n_{1}-1} & =0=x_{n_{1}+1},
\end{aligned}
$$

has a negative eigenvalue $\mu_{1}^{\left(n_{1}\right)}$ and its corresponding eigenfunction $\varphi^{\left(n_{1}\right)}$ with $\left\|\varphi^{\left(n_{1}\right)}\right\|=1$ can be chosen so that $\varphi_{i}^{\left(n_{1}\right)}>0$ for all $i \in\left[-n_{1}, n_{1}\right]$. For any $\varepsilon>0$, we define

$$
\underline{x}_{i}= \begin{cases}\varepsilon \varphi_{i}^{\left(n_{1}\right)}, & i \in\left[-n_{1}, n_{1}\right], \\ 0, & i \in \mathbb{Z} \backslash\left[-n_{1}, n_{1}\right] .\end{cases}
$$

At this time, we have

$$
\begin{gathered}
-\Delta^{2}\left(\varepsilon \varphi_{i-1}^{\left(n_{1}\right)}\right)-\lambda p_{i}\left(\varepsilon \varphi_{i}^{\left(n_{1}\right)}\right)+\lambda\left(\varepsilon \varphi_{i}^{\left(n_{1}\right)}\right)^{1+\alpha} \\
=\mu_{1}^{\left(n_{1}\right)}\left(\varepsilon \varphi_{i}^{\left(n_{1}\right)}\right)+\lambda\left(\varepsilon \varphi_{i}^{\left(n_{1}\right)}\right)^{1+\alpha} \leq 0
\end{gathered}
$$

for $i \in\left[-n_{1}, n_{1}\right]$ and sufficiently small $\epsilon$. When $i=n_{1}+1$,

$$
\begin{aligned}
-\Delta^{2} \underline{x}_{i-1} & =-\underline{x}_{n_{1}+2}+2 \underline{x}_{n_{1}+1}-\underline{x}_{n_{1}} \\
& =-\varepsilon \varphi_{n_{1}}^{\left(n_{1}\right)}<0
\end{aligned}
$$

and $-\Delta^{2} \underline{x}_{i-1} \equiv 0$ for $i>n_{1}+1$. Similarly, we can also prove that $-\Delta^{2} \underline{x}_{i-1} \leq 0$ for $i \leq-n_{1}-1$. Thus, the sequence $\underline{x}$ defined in (42) is a subsolution of (1).

Note that $\alpha>0$ and the sequence $\left\{p_{i}\right\}$ is bounded. Thus, the sufficiently large positive constant $M \geq \underline{x}$ is a supersolution of (1).

$$
\begin{aligned}
& \text { For } f\left(i, x_{i}\right)=\lambda\left(p_{i} x_{i}-x_{i}^{1+\alpha}\right) \text {, we have } \\
& f\left(i, s_{2}\right)-f\left(i, s_{1}\right) \\
& =\lambda\left(p_{i} s_{2}-s_{2}^{1+\alpha}\right)-\lambda\left(p_{i} s_{1}-s_{1}^{1+\alpha}\right) \\
& =\lambda\left(p_{i}-(1+\alpha) \xi^{\alpha}\right)\left(s_{2}-s_{1}\right),
\end{aligned}
$$


where $\xi \in\left(s_{1}, s_{2}\right)$. Note that the sequences $\left\{p_{i}\right\},\left\{\underline{x}_{i}\right\}$, and $M$ are bounded; thus, (1) satisfies condition (34). By Theorem 4, problem (1) has a solution $u$ such that $0 \leq \underline{x} \leq u \leq M$. We claim that $u>0$. Suppose there exists $i_{1} \in \mathbb{Z}$ such that $u_{i_{1}}=0$; then we obtain $u_{i_{1}-1}+u_{i_{1}+1}=0$ since $-\Delta^{2} u_{i_{1}-1}=\lambda\left(p_{i_{1}} u_{i_{1}}-\right.$ $\left.u_{i_{1}}^{1+\alpha}\right)$. Thus we obtain $u_{i_{1}-1}=u_{i_{1}+1}=u_{i_{1}}=0$. So $u \equiv 0$, which contradicts $u \neq \equiv$. The proof is complete.

Theorem 6. Assume that $\lambda>\lambda_{1}^{(\infty)}$ and there exists a constant $C>6 / \lambda$ and $n_{0}>\left|i_{0}\right|$ such that

$$
p_{i} \leq \begin{cases}\frac{C}{[i(i+1)]^{\alpha}}, & \alpha \in(0,1], \\ -\frac{C}{i(i+1)}, & \alpha>1\end{cases}
$$

for $|i|>n_{0}$. Then (1) has a positive soliton and there exists a constant $M>0$ and $n_{2} \geq n_{0}$ such that

$$
x_{i} \leq \frac{M}{i(i+1)} \quad \text { for }|i| \geq n_{2} .
$$

Proof. Let $\underline{x}$ be the subsolution of (1) obtained in Theorem 5 and

$$
\phi_{i}=\frac{M}{i(i+1)}, \quad i \neq 0, \pm 1, \pm 2,
$$

where $M>0$ is a constant determined later. By simple calculation, we have

$$
\begin{gathered}
\Delta \phi_{i-1}=\frac{M}{i(i+1)}-\frac{M}{i(i-1)}=-\frac{2 M}{i\left(i^{2}-1\right)}, \\
-\Delta^{2} \phi_{i-1}=\frac{2 M}{i(i+1)(i+2)}-\frac{2 M}{i\left(i^{2}-1\right)} \\
=-\frac{2 M}{i(i+1)} \cdot \frac{3}{(i-1)(i+2)}, \\
-\Delta^{2} \phi_{i-1}-\lambda p_{i} \phi_{i}+\lambda \phi_{i}^{1+\alpha} \\
=\frac{\lambda M}{i(i+1)}\left(\frac{-6}{\lambda(i-1)(i+2)}+\left(\frac{M}{i(i+1)}\right)^{\alpha}-p_{i}\right) .
\end{gathered}
$$

By the assumption on $p$, there exist $n_{2} \geq n_{0}$ and $M_{1}>0$ such that

$$
\frac{-6}{\lambda(i-1)(i+2)}+\left(\frac{M}{i(i+1)}\right)^{\alpha}-p_{i} \geq 0
$$

for $M \geq M_{1}$ and $|i| \geq n_{2}$.

For any $M \geq M_{1}$, define

$$
\psi_{i}= \begin{cases}\frac{M}{i(i+1)}, & |i| \geq n_{2}, \\ M, & |i|<n_{2} .\end{cases}
$$

We will show that $\psi$ is upper-solution of (1) by appropriate choice of $M$. In fact,

(a) for $|i|>n_{2}$, we have $-\Delta^{2} \psi_{i-1}-\lambda p_{i} \psi_{i}+\lambda \psi_{i}^{1+\alpha} \geq 0$ by the choice of $M$; (b) for $|i|<n_{2}-1$, we have

$$
-\Delta^{2} \psi_{i-1}-\lambda p_{i} \psi_{i}+\lambda \psi_{i}^{1+\alpha}=-\lambda p_{i} M+\lambda M^{1+\alpha} \geq 0
$$

by choosing $M>M_{1}$ large enough since $\alpha>0$ and $p$ is bounded;

(c) similarly, for $i=n_{2}, n_{2}-1,-n_{2},-n_{2}+1$, one can show that $-\Delta^{2} \psi_{i-1}-\lambda p_{i} \psi_{i}+\lambda \psi_{i}^{1+\alpha} \geq 0$ for $M>M_{1}$ large enough.

In view of (a)-(c), we construct upper-solution $\psi$ of (1) with $\psi \geq \underline{x}$. Using the sub- and upper-solution Theorem 4, we complete the proof.

Theorem 7. Assume that there exists a positive integer $m>\left|i_{0}\right|$ such that $p_{i} \leq 0$ for $|i| \geq m$. Let $x$ be a bounded positive solution of (1); then

$$
\lim _{|i| \rightarrow \infty} x_{i}=0 .
$$

Proof. At this time, we have

$$
-\Delta^{2} x_{i-1}=\lambda\left(p_{i} x_{i}-x_{i}^{1+\alpha}\right) \leq 0 \text { for }|i| \geq m .
$$

Hence $\Delta x_{i-1}$ is an increasing sequence for $|i| \geq m$ and so there exists a positive integer $n_{1}$ such that $\Delta x_{i}$ is one sign for $|i|>n_{1}$. Thus $x$ is eventually a monotone sequence for $|i|>n_{1}$. Note that $x$ is bounded. Thus, $\lim _{n \rightarrow+\infty} x_{n}$ and $\lim _{n \rightarrow-\infty} x_{n}$ exist.

If $\lim _{n \rightarrow+\infty} x_{n} \neq 0$, we have

$$
\begin{gathered}
\Delta^{2} x_{i-1} \geq \lambda x_{i}^{1+\alpha} \quad \text { for } i \geq m, \\
\Delta x_{n}-\Delta x_{n_{0}-1} \geq \lambda \sum_{i=n_{0}}^{n} x_{i}^{1+\alpha},
\end{gathered}
$$

which implies that

$$
\lim _{n \rightarrow+\infty} x_{n}=+\infty .
$$

This is impossible since $x$ is bounded and so we must have $\lim _{n \rightarrow+\infty} x_{n}=0$. Similarly, we also have $\lim _{n \rightarrow-\infty} x_{n}=0$. The proof is complete.

Theorem 8. For any $\lambda \neq 0$, there exists at most one positive solution of (1) such that $\lim _{|i| \rightarrow \infty} x_{i}=0$.

Proof. Suppose that $u$ and $v$ are such two distinct solutions. As before we can construct an arbitrarily small subsolution and so there must exist a solution $w$ of (1) such that $w \leq u$ and $w \leq v$. Multiplying the $u$-equation by $w$ and the $w$-equation by $u$, then we have

$$
\sum_{i=-n}^{n}\left(u_{i} \Delta^{2} w_{i-1}-w_{i} \Delta^{2} u_{i-1}\right)=\lambda \sum_{i=-n}^{n} u_{i} w_{i}\left(w_{i}^{\alpha}-u_{i}^{\alpha}\right)
$$

or

$$
\begin{gathered}
u_{n} \Delta w_{n}-u_{-n} \Delta w_{-n-1}-w_{n} \Delta u_{n}+w_{-n} \Delta u_{-n-1} \\
=\lambda \sum_{i=-n}^{n} u_{i} w_{i}\left(w_{i}^{\alpha}-u_{i}^{\alpha}\right) .
\end{gathered}
$$


Let $n \rightarrow+\infty$; we have

$$
\sum_{i=-\infty}^{\infty} u_{i} w_{i}\left(w_{i}^{\alpha}-u_{i}^{\alpha}\right)=0
$$

which implies that $u=w$. Similarly, we can also prove that $v=w$. The proof is complete.

Corollary 9. Assume that all conditions of Theorem 8 hold; then (1) has a unique positive soliton.

\section{Conclusion}

This paper studied a discrete logistic steady-state equation with both positive and negative birth rate of population. By using sub- and upper-solution method (Theorem 4), the existence of positive solution and positive soliton is obtained (Theorems 5 and 6). Uniqueness of homoclinic type solution is also obtained (Theorem 8 ).

\section{Appendix}

To obtain a positive eigenfunction of (13) or a positive eigenvector of (16), we need the following lemma. We give the proof by the method of [22].

Lemma A.1. For any $(b-a+1) \times(b-a+1)$ diagonal matrix

$$
Q=\left(\begin{array}{cccc}
q_{a} & 0 & \cdots & 0 \\
0 & q_{a+1} & \cdots & 0 \\
& \cdots & \cdots & \\
0 & \cdots & 0 & q_{b}
\end{array}\right), \quad q_{i} \in \mathbb{R}, i \in[a, b]
$$

let $\mu_{1}$ be the minimal eigenvalue of $A+Q$, where $A$ is defined in (14). Then $\mu_{1}$ is simple and the corresponding eigenvector $\varphi^{(1)}$ can be chosen to be positive.

Proof. Without loss of generality, we assume $q_{i}>0$ for $i \in$ $[a, b]$. If this is not the case, choose a constant $C>0$ large enough such that $q_{i}+C>0$. Consider the matrix $A+Q+C E$ instead, where $E$ is a $(b-a+1) \times(b-a+1)$ identity matrix. Denote $\widetilde{Q}=Q+C E$; then $\widetilde{q}_{i}>0$ for any $i \in[a, b]$.

Since $A+Q$ is a real $(b-a+1) \times(b-a+1)$ symmetric positive definite matrix, $A+Q$ has $b-a+1$ real eigenvalues. We repeat each eigenvalue according to its multiplicity as follows:

$$
0<\mu_{1} \leq \mu_{2} \leq \cdots \leq \mu_{b-a+1}
$$

and we choose eigenvectors $\varphi^{(1)}, \varphi^{(2)}, \ldots, \varphi^{(b-a+1)}$ for $A+Q$ such that

$$
\left\{\varphi^{(1)}, \varphi^{(2)}, \ldots, \varphi^{(b-a+1)}\right\}
$$

is an orthonormal basis for $\mathbb{R}^{b-a+1}$. In the following we give the proof of Lemma A.1 in four steps.
Step 1. For any $u, v \in \mathbb{R}^{b-a+1}$, define $B[u, v]=v^{T}(A+Q) u$. It is obvious that

$$
\begin{aligned}
& B\left[\varphi^{(i)}, \varphi^{(j)}\right]=\left\{\begin{array}{ll}
0, & i \neq j, \\
\mu_{i}, & i=j,
\end{array}, i, j \in[a, b],\right. \\
& \mu_{1}=\min \left\{B[u, u] \mid u \in \mathbb{R}^{b-a+1},\|u\|=1\right\} .
\end{aligned}
$$

Step 2. We claim that if $u \in \mathbb{R}^{b-a+1}$ and $\|u\|=1$, then $u$ is a solution of

$$
(A+Q) u=\mu_{1} u
$$

if and only if

$$
B[u, u]=\mu_{1} .
$$

Obviously (A.5) implies (A.6). On the other hand, suppose (A.6) is valid. Then, writing $d_{k}=\left(u, \varphi^{(k)}\right)$, we have

$$
\begin{gathered}
u=\sum_{k=1}^{b-a+1}\left(u, \varphi^{(k)}\right) \varphi^{(k)}=\sum_{k=1}^{b-a+1} d_{k} \varphi^{(k)}, \\
\sum_{k=1}^{b-a+1} d_{k}^{2} \mu_{1}=\mu_{1}=B[u, u]=\sum_{k=1}^{b-a+1} d_{k}^{2} \mu_{k} .
\end{gathered}
$$

Hence

$$
\sum_{k=1}^{b-a+1}\left(\mu_{k}-\mu_{1}\right) d_{k}^{2}=0
$$

Consequently $d_{k}=\left(u, \varphi^{(k)}\right)=0$, if $\mu_{k}>\mu_{1}$. It follows that

$$
u=\sum_{k=1}^{m}\left(u, \varphi^{(k)}\right) \varphi^{(k)}
$$

for some $m$, where $(A+Q) \varphi^{(k)}=\mu_{1} \varphi^{(k)}$ for $k=1,2, \ldots, m$. Therefore

$$
(A+Q) u=\sum_{k=1}^{m}\left(u, \varphi^{(k)}\right)(A+Q) \varphi^{(k)}=\mu_{1} u .
$$

This proves (A.5).

Step 3. We will show that if $u \in \mathbb{R}^{b-a+1}$ satisfies $(A+Q) u=$ $\mu_{1} u, u \neq 0$, then either $u>0$ or $u<0$.

To see this, let us assume without loss of generality that $\|u\|=1$ and note $\alpha+\beta=1$ for $\alpha=\left\|u^{+}\right\|^{2}, \beta=\left\|u^{-}\right\|^{2}$, where $u_{i}^{+}=\max \left\{u_{i}, 0\right\}$ and $u_{i}^{-}=\max \left\{-u_{i}, 0\right\}$. In this case, we have

$$
\begin{aligned}
\mu_{1} & =B[u, u]=B\left[u^{+}, u^{+}\right]+B\left[u^{-}, u^{-}\right] \\
& \geq \mu_{1}\left\|u^{+}\right\|^{2}+\mu_{1}\left\|u^{-}\right\|^{2}=\mu_{1} .
\end{aligned}
$$

Thus

$$
B\left[u^{+}, u^{+}\right]=\mu_{1}\left\|u^{+}\right\|^{2}, \quad B\left[u^{-}, u^{-}\right]=\mu_{1}\left\|u^{-}\right\|^{2} .
$$


By Step $2, u^{+}$and $u^{-}$satisfy $(A+Q) u^{+}=\mu_{1} u^{+},(A+Q) u^{-}=$ $\mu_{1} u^{-}$. Thus $(A+Q) u^{+} \geq 0,(A+Q) u^{-} \geq 0$. That is,

$$
\begin{aligned}
& 2 u_{a}^{+}-u_{a+1}^{+}+q_{a} u_{a}^{+} \geq 0 \\
& -u_{a}^{+}+2 u_{a+1}^{+}-u_{a+2}^{+}+q_{a+1} u_{a+1}^{+} \geq 0 \\
& -u_{a+1}^{+}+2 u_{a+2}^{+}-u_{a+3}^{+}+q_{a+2} u_{a+2}^{+} \geq 0 \\
& \ldots \quad \ldots \\
& -u_{b-1}^{+}+2 u_{b}^{+}+q_{b} u_{b}^{+} \geq 0 .
\end{aligned}
$$

If there exists an $i_{0} \in[a, b]$ such that $u_{i_{0}}^{+}=0$, then $u^{+}=$ 0 . Similar arguments apply to $u^{-}$, and so either $u>0$ or $u<0$. Thus, we can choose a positive eigenvector $\varphi^{(1)}$ corresponding to the minimal eigenvalue $\mu_{1}$.

Step 4. Finally assume that $u$ and $\tilde{u}$ are two eigenvectors corresponding to $\mu_{1}$; in view of Step 3 ,

$$
\sum_{i=a}^{b} u_{i} \neq 0, \quad \sum_{i=a}^{b} \tilde{u}_{i} \neq 0
$$

and so there exists a real constant $k$ such that

$$
\sum_{i=a}^{b} u_{i}=k \sum_{i=a}^{b} \widetilde{u}_{i}
$$

or

$$
\sum_{i=a}^{b}\left(u_{i}-k \tilde{u}_{i}\right)=0 .
$$

But since $u-k \tilde{u}$ also satisfies $(A+Q)(u-k \widetilde{u})=\mu_{1}(u-k \tilde{u})$, by Step $3, u-k \tilde{u}=0$ or $u-k \tilde{u}>0$ or $u-k \tilde{u}<0$. In view of (A.16), we have $u-k \tilde{u}=0$. Hence the eigenvalue $\mu_{1}$ is simple. This completes the proof of Lemma A.1.

\section{Conflict of Interests}

The authors declare that there is no conflict of interests regarding the publication of this paper.

\section{Acknowledgment}

The authors would like to thank the anonymous referees for their comments and suggestions on the paper.

\section{References}

[1] R. S. MacKay and S. Aubry, "Proof of existence of breathers for time-reversible or Hamiltonian networks of weakly coupled oscillators," Nonlinearity, vol. 7, no. 6, pp. 1623-1643, 1994.

[2] G. Arioli and F. Gazzola, "Periodic motions of an infinite lattice of particles with nearest neighbor interaction," Nonlinear Analysis: Theory, Methods \& Applications, vol. 26, no. 6, pp. 1103-1114, 1996.
[3] V. Koukouloyannis and S. Ichtiaroglou, "Existence and stability of breathers in lattices of weakly coupled two-dimensional near-integrable Hamiltonian oscillators," Physica D: Nonlinear Phenomena, vol. 201, no. 1-2, pp. 65-82, 2005.

[4] S. Aubry, G. Kopidakis, and V. Kadelburg, "Variational proof for hard discrete breathers in some classes of Hamiltonian dynamical systems," Discrete and Continuous Dynamical Systems Series $B$, vol. 1, no. 3, pp. 271-298, 2001.

[5] Z. Zhou and J. Yu, "On the existence of homoclinic solutions of a class of discrete nonlinear periodic systems," Journal of Differential Equations, vol. 249, no. 5, pp. 1199-1212, 2010.

[6] Z. Zhou, J. Yu, and Y. Chen, "On the existence of gap solitons in a periodic discrete nonlinear Schröinger equation with saturable nonlinearity," Nonlinearity, vol. 23, no. 7, pp. 1727-1740, 2010.

[7] Z. Zhou, J. Yu, and Y. Chen, "Homoclinic solutions in periodic difference equations with saturable nonlinearity," Science China Mathematics, vol. 54, no. 1, pp. 83-93, 2011.

[8] G. Chen and S. Ma, "Homoclinic orbits of superlinear Hamiltonian systems," Proceedings of the American Mathematical Society, vol. 139, no. 11, pp. 3973-3983, 2011.

[9] G. Chen and S. Ma, "Discrete nonlinear Schröinger equations with superlinear nonlinearities," Applied Mathematics and Computation, vol. 218, no. 9, pp. 5496-5507, 2012.

[10] J. Cuevas, P. G. Kevrekidis, D. J. Frantzeskakis, and B. A. Malomed, "Discrete solitons in nonlinear Schrödinger lattices with a power-law nonlinearity," Physica D: Nonlinear Phenomena, vol. 238, no. 1, pp. 67-76, 2009.

[11] G. James, "Centre manifold reduction for quasilinear discrete systems," Journal of Nonlinear Science, vol. 13, no. 1, pp. 27-63, 2003.

[12] A. Pankov and V. Rothos, "Periodic and decaying solutions in discrete nonlinear Schröinger with saturable nonlinearity," Proceedings of The Royal Society A: Mathematical, Physical and Engineering Sciences, vol. 464, no. 2100, pp. 3219-3236, 2008.

[13] M. Ma and Z. Guo, "Homoclinic orbits for second order selfadjoint difference equations," Journal of Mathematical Analysis and Applications, vol. 323, no. 1, pp. 513-521, 2006.

[14] M. J. Ma and Z. M. Guo, "Homoclinic orbits and subharmonics for nonlinear second order difference equations," Nonlinear Analysis. Theory, Methods \& Applications, vol. 67, no. 6, pp. 17371745, 2007.

[15] X. H. Tang and X. Lin, "Homoclinic solutions for a class of second-order Hamiltonian systems," Journal of Mathematical Analysis and Applications, vol. 354, no. 2, pp. 539-549, 2009.

[16] X. H. Tang, X. Lin, and L. Xiao, "Homoclinic solutions for a class of second order discrete Hamiltonian systems," Journal of Difference Equations and Applications, vol. 16, no. 11, pp. 12571273, 2010.

[17] G. A. Afrouzi, "On a nonlinear eigenvalue problem in ODE," Journal of Mathematical Analysis and Applications, vol. 303, no. 1, pp. 342-349, 2005.

[18] M. Bôcher, "The smallest characteristic numbers in a certain exceptional case," Bulletin of the American Mathematical Society, vol. 21, no. 1, pp. 6-9, 1914.

[19] Y. Shi and S. Chen, "Spectral theory of second-order vector difference equations," Journal of Mathematical Analysis and Applications, vol. 239, no. 2, pp. 195-212, 1999.

[20] Y. Shi and S. Chen, "Spectral theory of higher-order discrete vector Sturm-Liouville problems," Linear Algebra and its Applications, vol. 323, no. 1-3, pp. 7-36, 2001. 
[21] H. Sun and Y. Shi, "Eigenvalues of second-order difference equations with coupled boundary conditions," Linear Algebra and Its Applications, vol. 414, no. 1, pp. 361-372, 2006.

[22] L. C. Evans, Partial Differential Equations, vol. 2 of Graduate Studies in Mathematics, American Mathematical Society, 1998. 


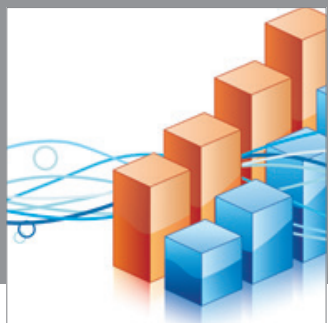

Advances in

Operations Research

mansans

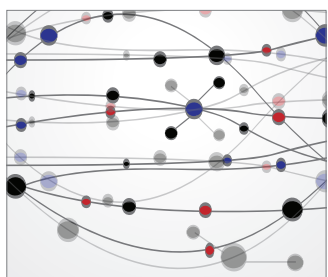

The Scientific World Journal
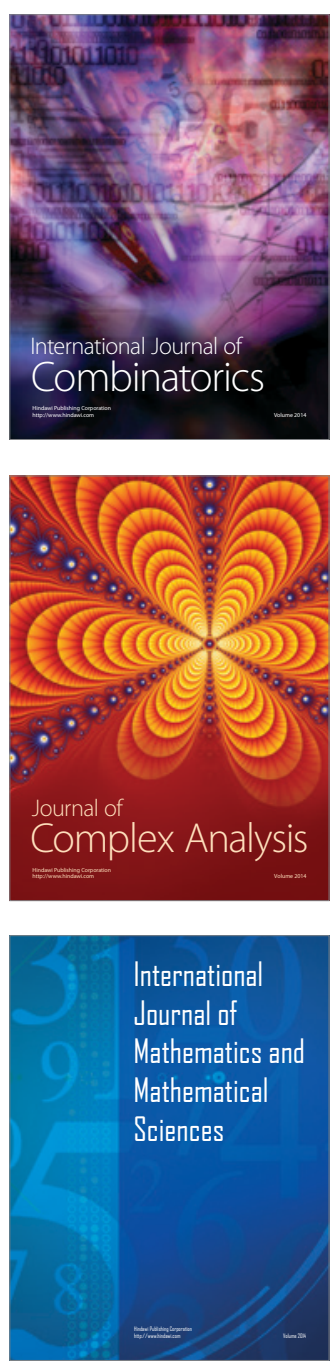
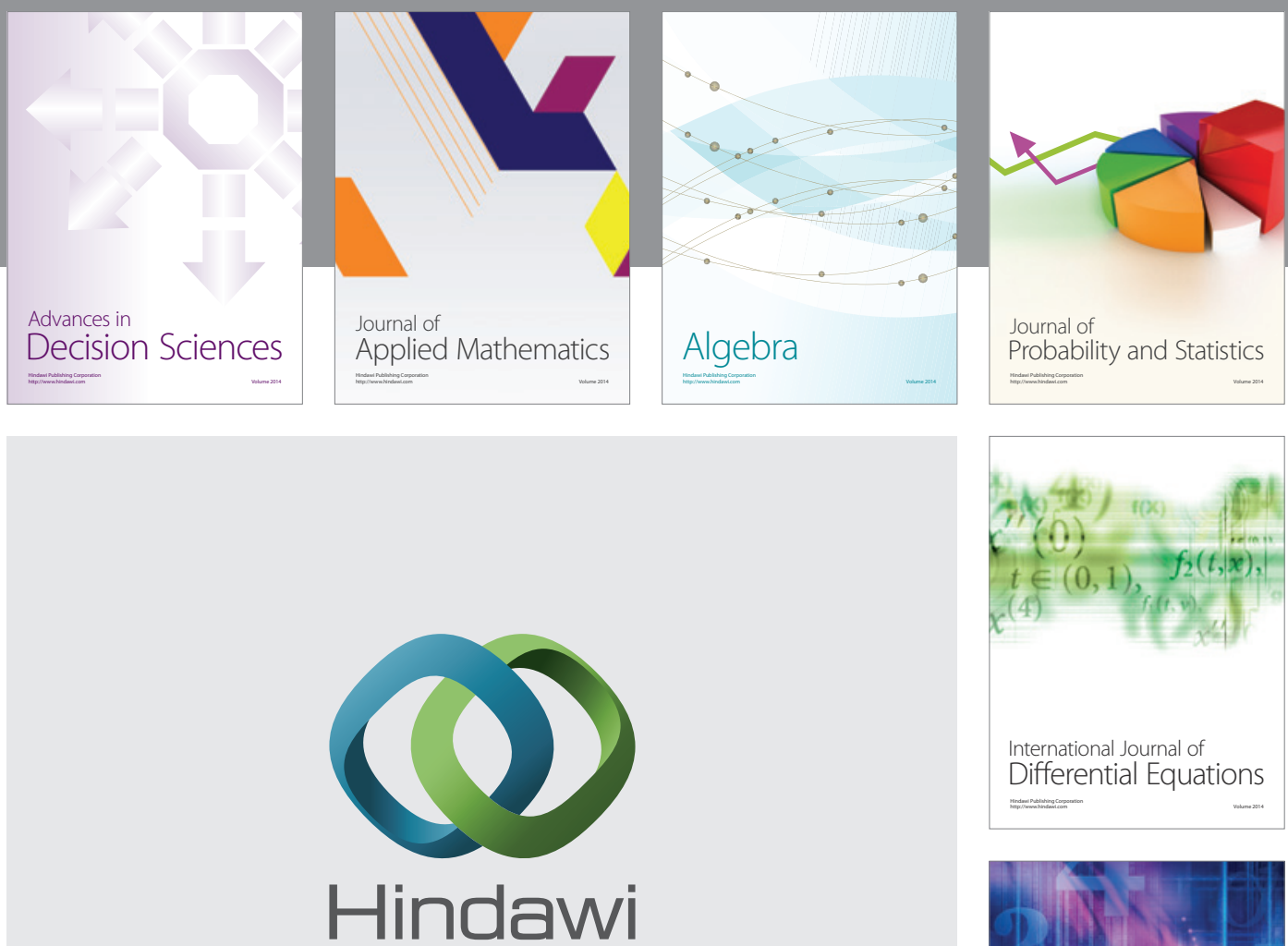

Submit your manuscripts at http://www.hindawi.com
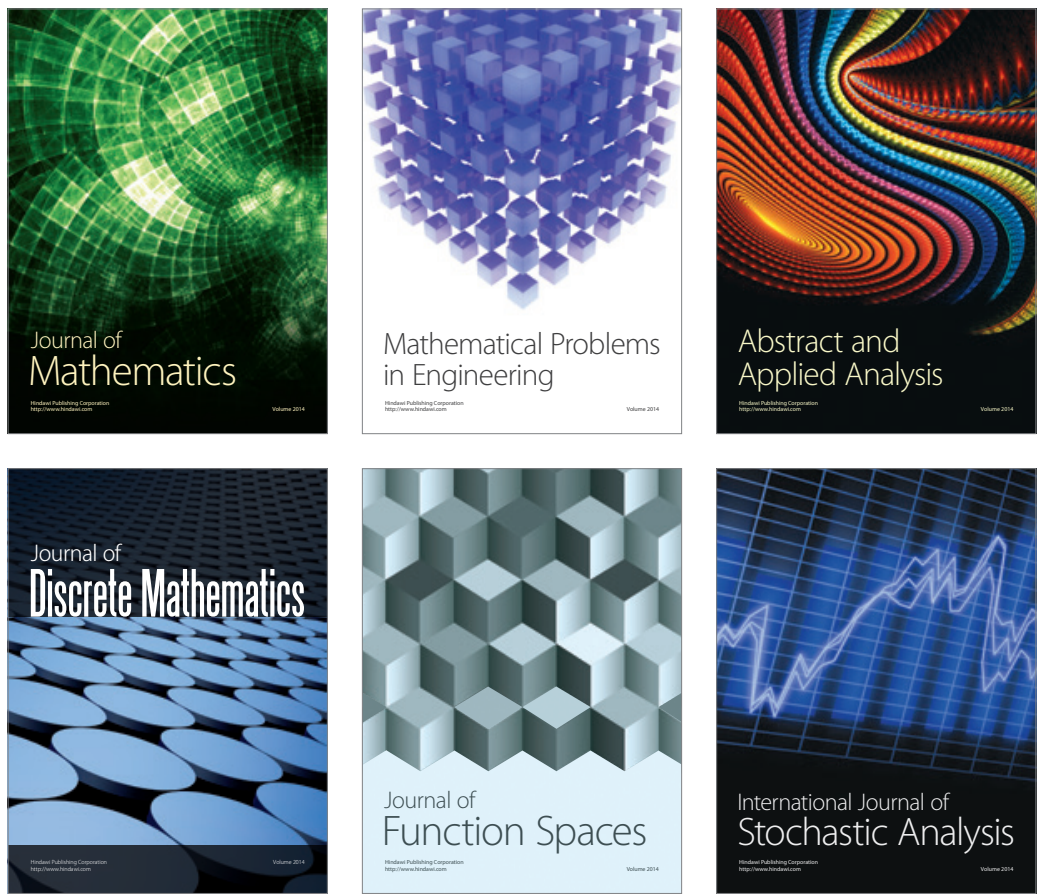

Journal of

Function Spaces

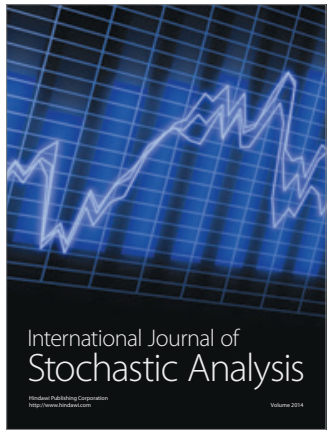

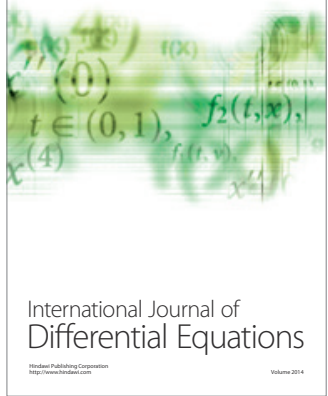
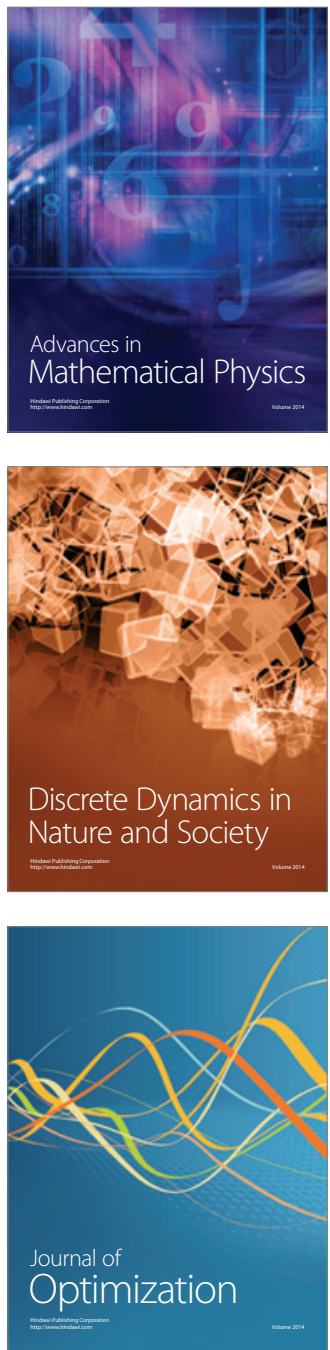